\title{
Multimodal deep representation learning for protein interaction identification and protein family classification
}

\author{
Da Zhang ${ }^{*}$ and Mansur Kabuka \\ From IEEE International Conference on Bioinformatics and Biomedicine (2018) \\ Madrid, Spain. 3-6 December 2018
}

\begin{abstract}
Background: Protein-protein interactions(PPIs) engage in dynamic pathological and biological procedures constantly in our life. Thus, it is crucial to comprehend the PPIs thoroughly such that we are able to illuminate the disease occurrence, achieve the optimal drug-target therapeutic effect and describe the protein complex structures. However, compared to the protein sequences obtainable from various species and organisms, the number of revealed protein-protein interactions is relatively limited. To address this dilemma, lots of research endeavor have investigated in it to facilitate the discovery of novel PPIs. Among these methods, PPI prediction techniques that merely rely on protein sequence data are more widespread than other methods which require extensive biological domain knowledge.

Results: In this paper, we propose a multi-modal deep representation learning structure by incorporating protein physicochemical features with the graph topological features from the PPI networks. Specifically, our method not only bears in mind the protein sequence information but also discerns the topological representations for each protein node in the PPI networks. In our paper, we construct a stacked auto-encoder architecture together with a continuous bag-of-words (CBOW) model based on generated metapaths to study the PPI predictions. Following by that, we utilize the supervised deep neural networks to identify the PPIs and classify the protein families. The PPI prediction accuracy for eight species ranged from $96.76 \%$ to $99.77 \%$, which signifies that our multi-modal deep representation learning framework achieves superior performance compared to other computational methods.
\end{abstract}

Conclusion: To the best of our knowledge, this is the first multi-modal deep representation learning framework for examining the PPI networks.

Keywords: Protein-protein interaction network, Multimodal deep neural network, Knowledge graph representation learning

\section{Backgrounds}

Protein-protein interaction (PPI) networks are becoming increasingly crucial for analyzing biomedical functions, retrospecting species evolution and analyzing different compounds that cause diseases. Moreover, comprehending the intrinsic patterns behind PPI networks facilitates the understanding of cancer-related protein-protein interfaces and the topological structures of the cancer

\footnotetext{
*Correspondence: zhang.1855@miami.edu

Department of Electrical and Computer Engineering, University of Miami, Coral Gables, FL, U.S.
}

networks. Normally, two groups of research methods can be formulated when analyzing PPI networks: computational biology methods and high-throughput experimental methods. Given a PPI network, computational biology methods calculate the distances between proteins according to network theory metrics (e.g. betweenness, centrality, average degree) or machine learning algorithms[1-3]. High-throughput techniques, on the contrary, including yeast two-hybrid screens (Y2Hs)[4], mass spectrometry protein complex identification (MS-PCI) [5] and Nuclear Magnetic Resonance (NMR)[6], etc. pro- 
duce large amounts of data for constructing primary protein databases. These databases provide primary and rich sources for developing molecular and functional networks. Nevertheless, these genome-based techniques demand expensive wet-lab investment and exhaustive lab work. Also, because of the equipment biases in the experimental environment, the results generated by these genome-based methods are subjected to inevitable inaccuracy. Moreover, compared with the significant amount of protein sequence data, the functional units that have been discovered are comparatively restricted. Previously, traditional machine learning algorithms such as decision trees (DT), naive bayes (NB) and nearest neighbor (NN)[7] have been utilized efficiently in lots of data mining tasks. Yet, these traditional machine learning techniques lack the capacity of discovering hidden associations and extracting discriminant features from the input complex data. Lately, accompanied with the advancement of AI techniques, deep learning methodologies[8] extracting non-linear and high dimensional features from the protein sequences $[9,10]$ have emerged as a new tendency. These deep learning techniques and frameworks have been recently applied in tremendous biomedical research fields, biological network analysis, and medical image examination. However, since natural and real-world data distributions are highly complex and multimodal, it is essential to incorporate different modalities and patterns from the data to attain satisfactory performance. Additionally, discovering biological pattern from the graph topology of these protein networks is fundamental in comprehending the functions of the cells and their constitutional proteins. When applying deep learning techniques to biological network analysis, these modalities include topological similarities such as $1 s t$-order similarity, $2 n d$-order similarity, and homology features extracted from protein sequences. Additionally, next-generation sequencing technologies also generate large amounts of DNA/RNA sequences which are then translated into protein peptides in the form of stacked amino acid residues. These protein sequences consist of fundamental molecules which perform biological functions for various species [11-13]. Thus, the functionality of a protein is encoded in the amino acid residues. To recognize the protein functionalities, researchers categorize proteins into various families such that proteins within the same family share similar functions or become the parts on the same pathway. In this paper, we propose a advanced multi-modal deep representation learning framework preserving different modalities to harvest both protein sequence similarity and topological proximity. This framework leverages both relational and physicochemical information from proteins and successfully integrates them using a late feature fusion technique. These concatenated features are provided to the interaction identifier and protein family classifier for the training and testing tasks.

To the best of our knowledge, this is the first multimodal deep representation learning framework for analyzing protein-protein interaction networks. Specifically, the contributions of our method are listed as follows:

- A novel multi-modal deep representation learning framework is presented that integrates both unsupervised learning and supervised learning to predict Protein-protein interactions and identify protein families.

- In the unsupervised learning phase, we integrate the multi-modality features learned from Continuous Bag of Word (CBOW) model based on generated metapaths and a stacked auto-encoder (SAE) model to combine topological proximity features and the physicochemical sequence features for each protein. The SAE model is effective when denoising the systems and is capable of reconstructing useful representations from the partial raw data.

- In the supervised learning phase, we feed the output from the unsupervised model into the supervised model and achieve the higher PPI prediction accuracy and protein family classification accuracy. We apply our model on the DIP and the HPRD networks to formulate low-dimensional representations for high-level protein features.

The remainder of the paper is organized as follows. We present the data preprocessing strategies, theoretical background and algorithms of our methods in the "Methods" section. The "Results" section describes the framework parameter settings, dataset statistics, and experimental results. Finally, we conclude the paper and envision the future work in the conclusion part.

\section{Methods}

In this section, we illustrate our proposed framework which can be divided into three phases including a protein sequence preprocessing phase, an unsupervised learning phase, and a supervised learning phase. Comprehensive illustrations of each phase associated with their inputs and outputs are examined in the following sections.

\section{Protein sequence preprocessing phase}

For computational intelligent machine learning and data mining methods, it is demanded that the lengths of the feature dimensions are the same. Consequently, encoding protein sequences with various length amino acids into equivalent length feature vectors are necessary for the following machine learning tasks. Therefore, in this phase, we extract physicochemical information from the protein residues consisting of stacked amino acids and transform them into equal length numerical vectors. In this 
procedure, we maintain the constitutional protein residue information as much as possible by obtaining the inherent information in the protein peptides. We use the following four methods for converting various lengths protein sequences into fixed length numerical vectors[14].

\section{Amino Acid Composition}

The amino acid composition(AAC) statistics is the proportion of each amino acid type inside a protein sequence. The AAC computes the ratio of each type of amino acid and convert the peptides into equal length numerical vectors. The AAC can be computed as follows:

$$
f r(t)=\frac{N(t)}{N}, t \in\{A, C, D, \ldots Y\}
$$

Here, $N(t)$ is the number of amino acid type $t$ in a protein sequence with length $N$ and $\{A, C, D, \ldots Y\}$ represents twenty types of amino acids.

\section{Grouped Amino Acid Composition(GAAC)}

For the Grouped Amino Acid Composition, the 20 types of amino acids are classified into five categories according to their physicochemical properties[15]. These five categories include the aliphatic group (g1: GAVLMI), aromatic group (g2: FYW), positive charge group (g3: KRH), negative charged group (g4: DE) and uncharged group (g5: STCPNQ)[14]. GAAC computes the frequency of each group of amino acids as follows:

$$
\begin{array}{r}
f(g)=\frac{N_{g}}{N}, g \in\{g 1, g 2, g 3, g 4, g 5\} \\
N\left(g_{t}\right)=\sum N(t), t \in\{g\}
\end{array}
$$

Here, $N_{g}$ is the number of amino acids in group $g, N(t)$ is the number of amino acid for type $t$, and $N$ is the total length of the peptide sequence.

\section{Conjoint Triad}

The Conjoint Triad(CT) takes into account the properties of one amino acid and its adjacent amino acids by considering three adjoining amino acids as an individual feature[16]. We first represent the protein sequence using a binary space $(V, F)$. For the amino acids that have been categorized into 7 classes[16], the length of $V$ can be computed as $7 \times 7 \times 7=343$. Therefore, the dimension of vector $V$ is 343 . Each cell $V_{i} \in V$ indicates a triad feature. $F$ is the number of vectors corresponding to $V . f_{i}$ is the value of the $i$ th dimension of $F$ representing the number of types $V_{i}$ appearing in the protein sequence. Therefore, the $C T$ descriptor for a protein sequence can be derived as follows:

$$
d_{i}=\frac{f_{i}-\min \left\{f_{1}, f_{2}, \ldots, f_{343}\right\}}{\max \left\{f_{1}, f_{2}, \ldots f_{343}\right\}}
$$

Here, $d_{i}$ is the normalization of $f_{i}$.

\section{Quasi-Sequence-Order}

For each amino acid type, a quasi-sequence-order descriptor can be defined as the following equation:

$$
X_{r}=\frac{f_{r}}{\sum_{1}^{20} f_{r}+w \sum_{d=1}^{\text {nlag }} \tau_{d}}, r=1,2, \ldots, 20
$$

Here, $f_{r}$ is the normalized occurrence of amino acid type $r$ and $w$ is the weighting factor initialized at $w=0.1[14]$. nlag is the maximal value of the looking back parameter lag. $\tau_{d}=\sum_{i=1}^{N-d}\left(d_{i, i+d}\right)^{2}, d=1,2,3, \ldots$, nlag, and $d_{i, i+d}$ is the distance between cell $d_{i}$ and $d_{i+d}$ given a distance matrix. In the experiments, we set the default value of the $\operatorname{lag}$ at 30.

\section{Unsupervised Learning Phase}

After preprocessing the raw sequential data, we transform various lengths of protein sequences into 468 equal length vectors using iFeature APIs [14]. In the unsupervised learning phase, we first extract the deep features from previously generated equal length vectors, which will be fed into supervised prediction model.

\section{Deep Feature Extraction}

To obtain the deep features from the 468 dimensional vectors, we utilize the Stacked Auto Encoder(SAE) framework as shown in Fig. 1 and in Eq. 6.

$$
\operatorname{Map}_{s e q}\left(\mathbf{p}_{i}\right)=\mathbf{v}_{i}
$$

First, the input layer in the SAE takes the protein $p_{i}$ 's feature vector $\mathbf{v}_{i}$ as the input vector generated during the data preprocessing phase. After $e$ intermediate encoding layers, we obtain the output vector $\mathbf{h}^{\text {out }}$ as shown in Eq. 7 from the output layer. Here, $\mathbf{W}_{e n c}^{k} \in \mathbb{R}^{n \times d_{k}}$ and bias bnc $_{\text {en }} \in \mathbb{R}^{d_{k}}$ are the weight matrix and bias vector for the $k t h$ hidden layer in the encoding layers and $\delta$ represents the output activation function. $e$ denotes the number of encoding layers. The output deep representation vector $\mathbf{h}^{\text {out }} \in \mathbb{R}^{d_{\text {out }}}$ of the input vector $\mathbf{v}_{i}$ is then projected back to the space of $\mathbf{v}_{i}$ using the decoding function through decoding layers. $\mathbf{W}_{d e c}^{k}$ is the weight matrix for the $k$ th decoding layer and $d$ represents the number of decoding hidden layers. In our model, we choose the number of encoding layers the same as the number of decoding layers, i.e. $e=d$. Also, the number of hidden units in encoding layers equals to the number of hidden units in the decoding layers. During training phase, we update the parameters by using Stochastic Gradient Descent (SGD) methods to minimize the $L_{2}$ loss function defined in Eq. 8. The whole deep feature extraction process can be depicted in Eq. 6, 7 and Algorithm 1.

$$
\begin{aligned}
\mathbf{h}^{\text {out }} & =\delta\left(\mathbf{W}_{\text {enc }}^{k} \mathbf{v}_{i}+\mathbf{b i a s}_{\text {enc }}\right), k=1,2,3 \ldots e \\
\hat{\mathbf{v}}_{i} & =\delta\left(\mathbf{W}_{\text {dec }}^{k} \mathbf{h}^{\text {out }}+\mathbf{b i a s}_{\text {dec }}\right), k=1,2,3 \ldots d
\end{aligned}
$$




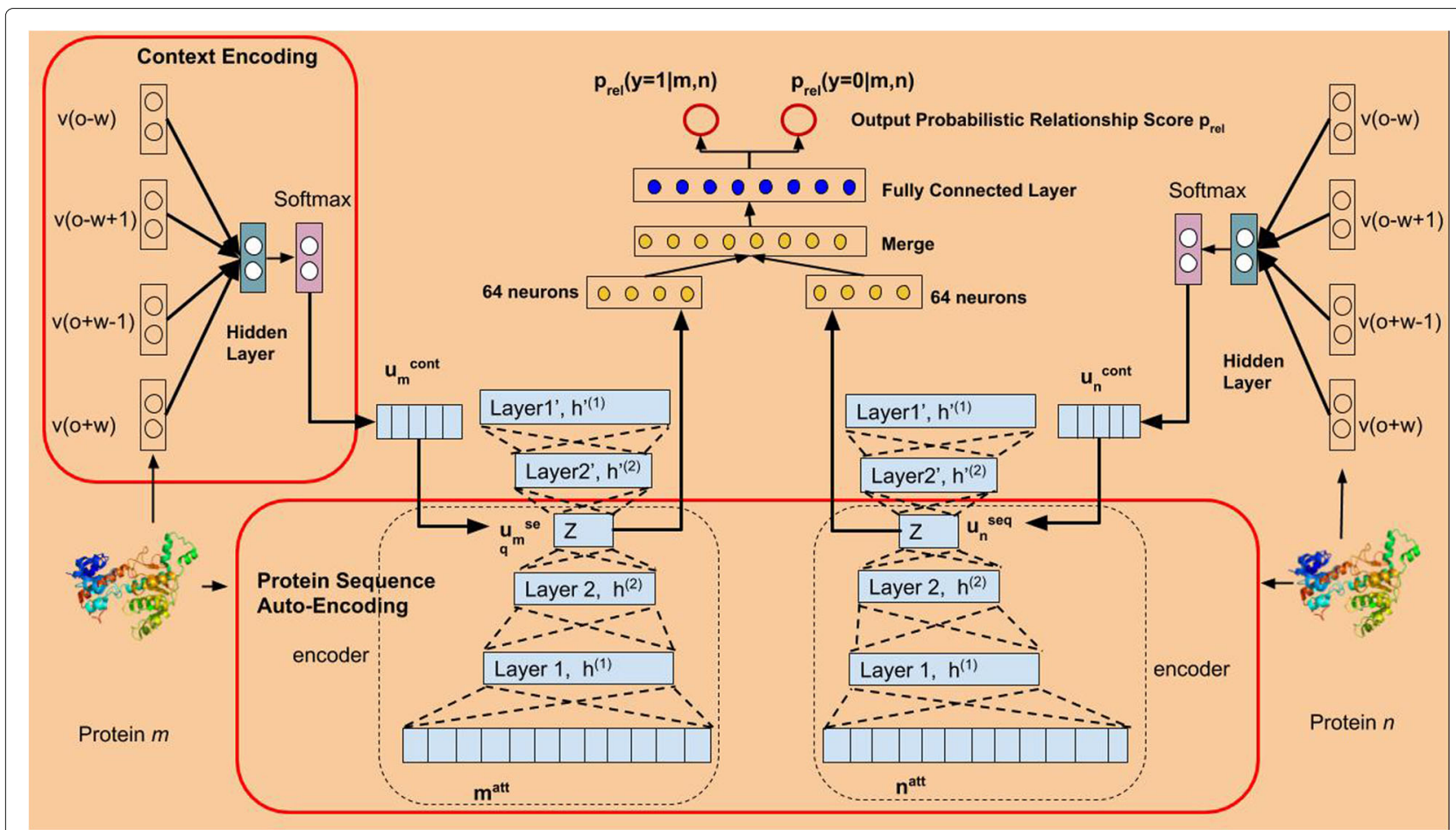

Fig. 1 Multi-modal Deep Representation Learning Framework

$$
L_{2}\left(\mathbf{v}_{i}, \hat{\mathbf{v}}_{i}\right)=\left(\mathbf{v}_{i}-\hat{\mathbf{v}}_{i}\right)^{2}=\left(\mathbf{v}_{i}-\left(\mathbf{W}_{d e c} \mathbf{h}+\mathbf{b i a s}_{d e c}\right)\right)^{2}
$$

\section{CBOW Model Based On Metapaths}

The CBOW(Continuous Bag of Words) model from natural language processing(NLP) techniques approximates the conditional probability in Eq. 9 [17]. Solving the optimization problem from Eq. 9 learns the distributive vectors that capture the proximity in the local network topology between nodes within the path length $w$ as shown in Fig. 2. The objective function we try to minimize can be described in Eq. 9. In this paper, for a center node $e_{c}$, we set $w=1$. Particularly, we only use the adjacent neighbors of node $e_{c}$ as contextual nodes to maximize the structural context-local proximity. Here, the total number of protein nodes in the network is represented by $N$ in Eq. 9. In the protein network, given a path consisting of protein nodes, $e_{1} \rightarrow e_{2} \rightarrow e_{3} \rightarrow \cdots \rightarrow e_{l}$, we adopt CBOW model to minimize the negative log-likelihood function in Eq. 9. In our method, we define the CBOW model as the unsupervised model since we did not label the nodes manually. Instead, the system will automatically learn the representation vectors of each node.

$$
L(\theta)=-\frac{1}{N} \sum_{n=1}^{N} \sum_{\substack{w \leq 0 \leq w \\ w \neq 0}} p\left(\mathbf{e}_{c} \mid \mathbf{e}_{c+o} ; \theta\right)
$$

\section{Homogeneous Metapaths Generation}

Recently, thanks to the scalability and adaptability of random walk technique, lots of research methods utilize random walk based methods to learn node representations over graph structured data [17-19]. Among these methods, metapath is the most recent one. During metapaths generation process, we set the length parameter as length to indicate the walking distance starting from each

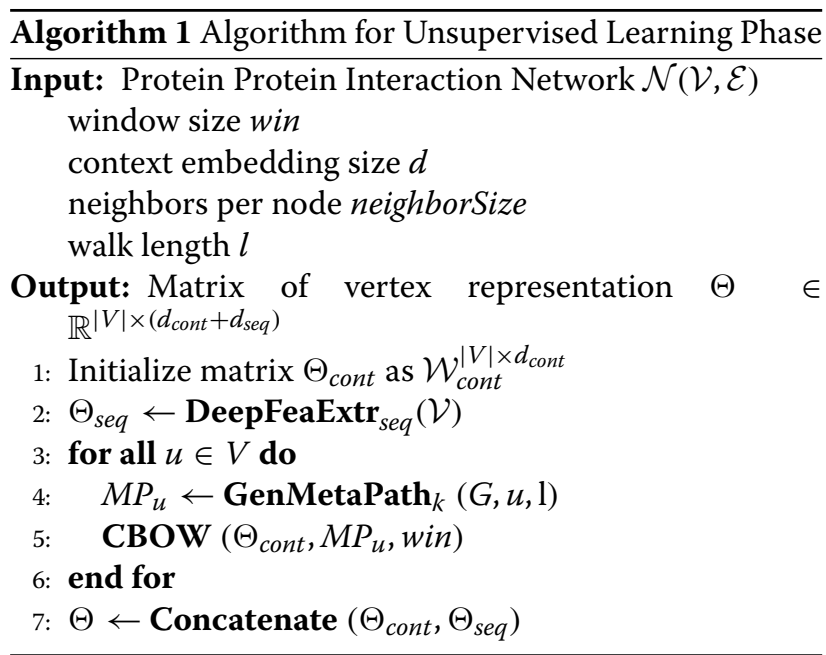




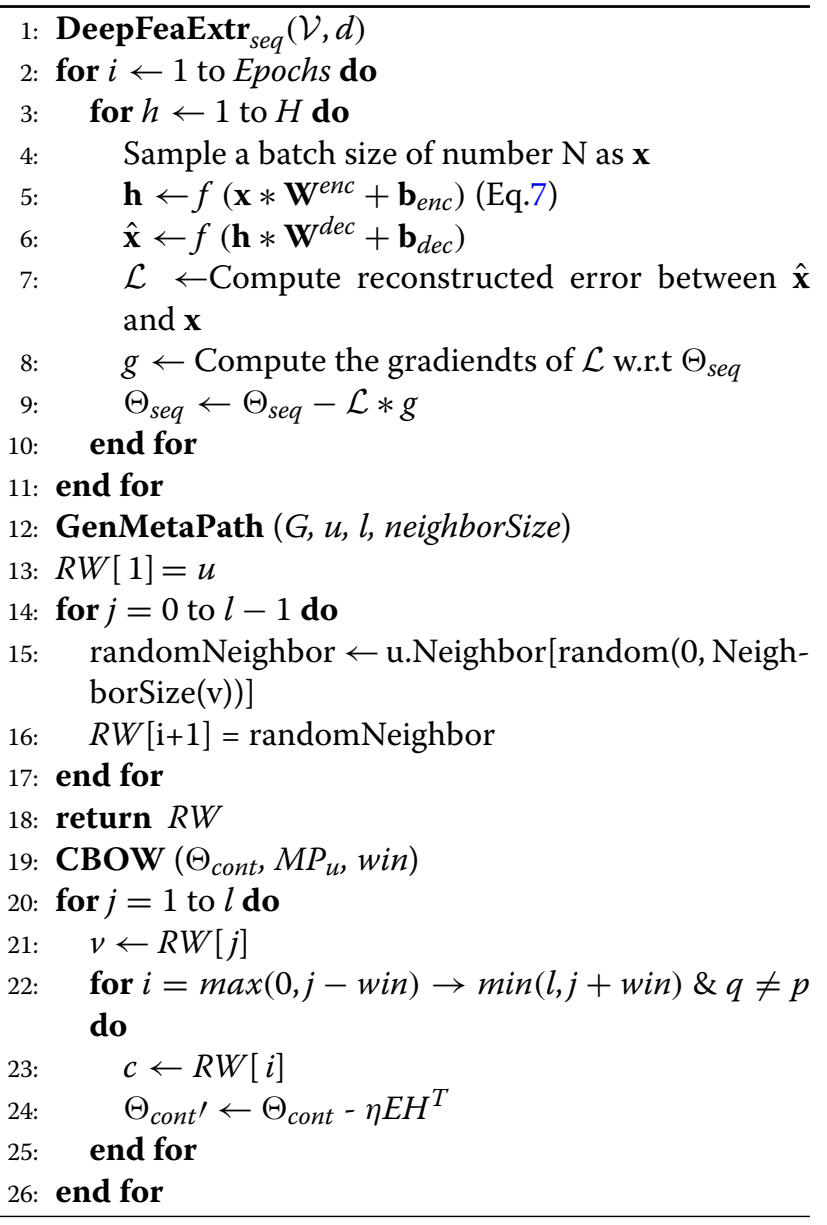

protein node in the network. Also, for each protein node, we set the neighborSize as the contextual sampling parameters indicating how many neighbors we take into account as shown in Fig. 2. After that, we apply CBOW model trying to learn the distributed node representations within the network structured data and maximizes the likelihood of preserving the topological similarities between nodes. We can regard metapath-based methods as a graph representation model that estimates the occurrence likelihood of observing $v_{i}$ given all the preceding vertices along the short path as shown in Eq. 10.

$$
\operatorname{Pr}\left(v_{j} \mid v_{i-w}, \ldots, v_{i+w}\right)
$$

The metapath approach presumes that within a network, the nodes co-occur along a short path tend to have intrinsic relationships. Therefore, based on random walk statistics [20,21], metapath-based methods optimize the node embeddings such that nodes have similar representations if they co-occur on short random walks over the graph [22]. The basic idea of this set of approaches is to learn the encoding matrix such that the following equation is satisfied.

$$
\mathbf{E N C}_{p}\left(\mathbf{z}_{\mathbf{i}}, \mathbf{z}_{\mathbf{j}}\right)=\frac{e^{z_{i}^{T} z_{j}}}{\sum_{v_{k} \in \mathcal{V}} e^{z_{i}^{T} z_{k}}} \approx p_{\mathcal{G}, \mathcal{T}}\left(v_{j} \mid v_{i}\right)
$$

Here, $v_{j}$ is the next neighboring node of $v_{i} . \mathbf{E N C}_{p}\left(\mathbf{z}_{\mathbf{i}}, \mathbf{z}_{\mathbf{j}}\right)$ represents the statistical probability of $v_{j}$ given its neighboring node $v_{i}$ along the path $p$. Since we only have one type of relationship or edge in the PPI networks, our metapaths generation process was defined as the homogeneous metapaths generation.

In our paper, the PPI networks are undirected graphs with vertices $\mathcal{V}$ representing proteins and edges $\mathcal{E}$ representing interactions. Accordingly, we generate nodeoriented metapaths for the protein nodes in the PPI network first and then apply CBOW model to learn the distributed topological representations for each protein node. The details for the unsupervised learning phase can be found in Algorithm 1.

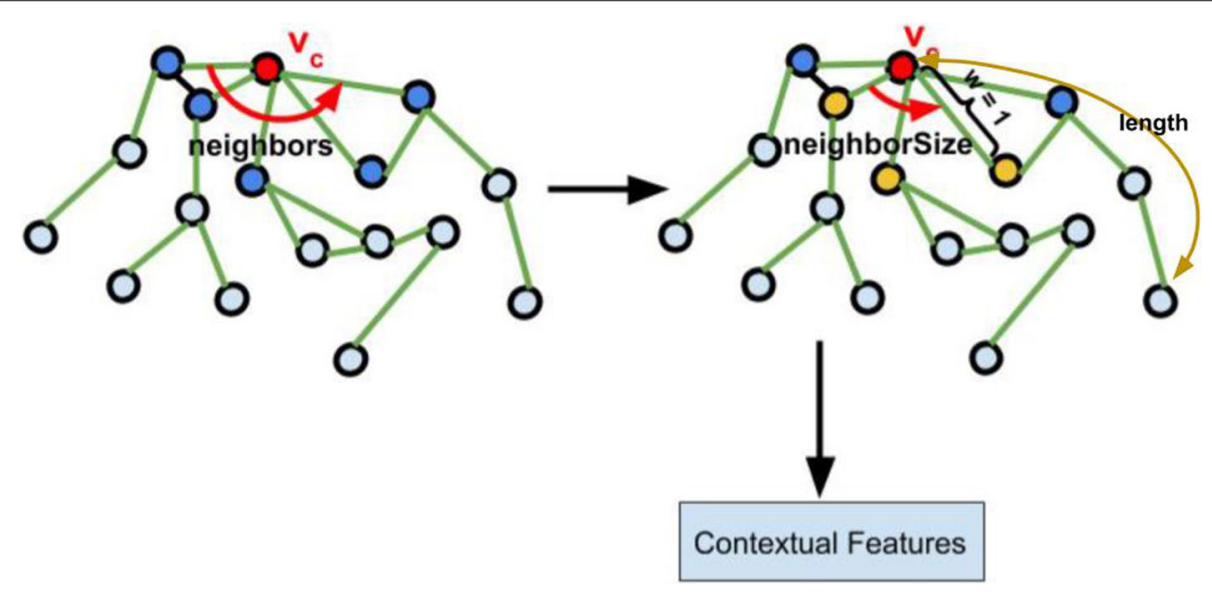

Fig. 2 Metapath Generation 


\section{Supervised Learning Phase}

After the unsupervised learning phase, deep protein features and protein topological representations are learned. We then employ the feature fusion for those extracted features before feeding them as the inputs to the supervised learning model.

\section{Feature Fusion}

Figures 1 and 3 present the integrated structure of our deep multi-modal representation learning framework, including the two phases of the leaning process. First, we fusion features learned from the CBOW model and SAE model. By doing this, various modalities as the outputs from the previous unsupervised learning phase are integrated.

$$
\mathbf{h}_{u}^{\text {out }}=\delta\left(\mathbf{W}\left[\begin{array}{c}
u_{p}^{s e q} \\
u_{p}^{\text {cont }}
\end{array}\right]+\mathbf{b}\right)
$$

As shown in Eq. 12, given a protein $p$, its topological feature is $u_{p}^{\text {cont }}$ and its deep protein sequence feature is $u_{p}^{\text {seq }}$ respectively. We concatenate these two features together as $u_{p}$ to represent $p$.

\section{Supervised Learning Model}

After we concatenate both topological proximity representations and deep physicochemical features of the proteins, we use them for the downstream protein interaction identification and protein multi-family classification tasks. Given two proteins $m$ and $n$, their deep sequence features are represented by vectors $u_{m}^{\text {seq }}$ and $u_{n}^{\text {seq }}$ respectively. Their topological proximity features are represented as $u_{m}^{\text {cont }}$ and $u_{n}^{\text {cont }}$, which are obtained by $C B O W$ model based on generated metapaths. For the $\mathrm{CBOW}$ model, we set the window size win $=1$ and the walking length as $l$. After this unsupervised learning process, we concatenate the obtained features as $u_{m}$ and $u_{n}$ and feed them into the supervised learning model. Then, the supervised learning model uses these features to perform PPI identification and Protein Multi-Family classification tasks as shown in Figs. 1 and 3.

- The PPI identification model consists of two deep neural networks separately as shown in Fig. 1. One is for protein $m$, and the other is for protein $n$. After the last layer, we combine the extracted lower dimensional features of the two proteins and feed those features into the fully connected layer connected to the output layer for classification. During the learning process, we use the binary cross entropy Eq. 13 as the loss function since the interaction can only exist or not. Therefore, the final classification results are the probability $\hat{y}=p(y \mid m, n)$ that the two given proteins $m$ and $n$ interact with each other.

$\mathcal{L}(\hat{y}, y)=-\frac{1}{N} \sum_{i=1}^{N}\left[y_{i} \log \hat{y}_{i}+\left(1-y_{i}\right) \log \left(1-\hat{y}_{i}\right)\right]$

- For classifying the protein families, we construct the model as shown in Fig. 3 and utilize the same features obtained from the unsupervised learning phase. We employ deep neural networks(DNN) for extracting non-linear hidden features. Since we are required to classify proteins into multiple categories, we use the categorical cross entropy Eq. 14 as the loss function here. Since this is multi-class classification task, we

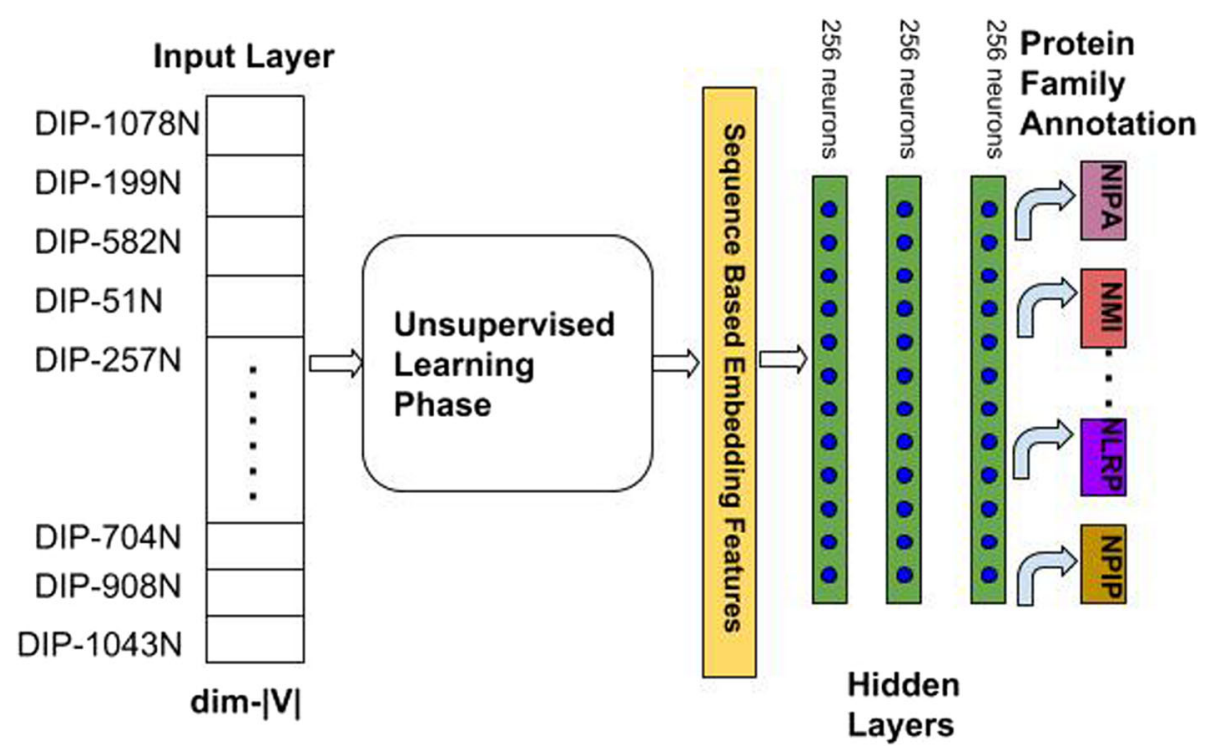

Fig. 3 Protein Family Classification Deep Neural Network 
calculate the individual loss rate for every class label $c$ per observation $o$ and sum the results over all of the $N$ training samples.

$$
\mathcal{L}=-\frac{1}{N} \sum_{i=1}^{N} \sum_{c=1}^{C} y_{o, c}\left[\log \left(\hat{y}_{o, c}\right)\right]
$$

During the experiments, we use stochastic gradient descent(SGD) as the optimization method.

\section{Results}

In the unsupervised learning phase, we set the the hidden layer parameters for the stacked auto-encoder(SAE) as $256-128-64-128-256.256,128,64,128$ and 256 represent the number of neurons for each hidden layer separately. During the SAE training phase, we use the mean squared error(MSE) as the loss function defined in Eq. 15. After the auto-encoding process, the protein sequence vectors are projected to the lower dimensional space with vector length 64 at the layer ' $z$ ' in Fig. 1. For the graph topology embedding, during the metapath generation process, we fix the contextual sampling parameter neighborSize $=4$ and the metapath length $l=10$ to generate the metapaths starting from each protein node. Then, we set the window size $\mathrm{win}=1$ and the node vector length $v=128$ for each protein such that the CBOW model is able to learn the distributive node representations.

$$
M S E=\frac{1}{n} \sum_{i=1}^{n}\left(Y_{i}-\hat{Y}_{i}\right)^{2}
$$

\section{Dataset Description}

During the experiments, we used two complete datasets including Database of Interacting Proteins (DIP) released 20170205_FULL dataset http://dip.mbi.ucla.edu/dip/ and Human Protein Reference Database http://www.hprd. org/ (HPRD), which are the benchmarks and complete databases most methods were tested on. The DIP dataset includes eight species D. melanogaster, S. cerevisiae, E. coli, C. elegans, H. sapiens, H. pylori, M. musculus, $R$. norvegicus. After removing the duplicate protein sequences and the self-interactions, we obtained 3790 PPIs for C.elegans, 22,067 for D. melanogaster, 11,521 for E.coli, 1358 for H.pylori, 6677 for H.apiens, 2385 for M.musculus, 523 for R.norgegicus and 22,502 for S.cerevisiae. First, we convert the raw protein data with various sequence lengths into 486 equal length vectors using the computational methods defined in the "Methods" section. Then, we generated the negative datasets from eight different subcellular locations including Cytoplasm, Nucleus, Endoplasmic reticulum, Golgi apparatus, lysosome, Mitochondrion, Cell Membrane and Lipid-ancho[10], in which different species of proteins reside. After that, we generated the corresponding negative samples by randomly matching those proteins with others found in the different subcellular locations. To avoid biased data, we generate the equal number of negative samples as the positive samples. The subcellular location information can be accessed from the UniProt database https://www.uniprot. org/locations/. After constructing the data, we mixed and shuffled the data for each species in the DIP dataset. Then, we split the data into the training dataset and testing dataset with the ratio of $80 \%$ and $20 \%$ respectively.

During the training phase, for each species, we used the PPIs in the training dataset to generate metapaths. The PPIs in the testing dataset are hold-out. We trained the CBOW model for 10 epochs and the SAE model for 50 epochs during the unsupervised learning phase. Figure 4 presents the MSE loss of training dataset and validation dataset for S.cerevisiae species during the training process using SAE framework. From the result, it can be seen that the validation loss and the training loss are synchronized with each other. This indicates that our model is not overfitting: the validation loss is decreasing instead of increasing, and there is rarely any gap between the training and validation loss. For the $\mathrm{CBOW}$ model, we give an example in Table 1 after we train with the H.sapiens dataset. The float values indicate the cosine similarity between the query protein node and the top-10 most similar protein nodes in descending order. Given a query protein with ID DIP- $41844 \mathrm{~N}$, which is the protein 5-hydroxytryptamine receptor $2 A$, we returned the most similar proteins measured by cosine similarity with respect to the query. The returning results can be verified by checking actual neighbors of DIP-41844N in the DIP database. It turns out that all the 1-hop neighbors of DIP-41844N have been correctly returned by the CBOW model ranked by their similarity scores. After the unsupervised learning phase, we performed late fusion on these deep abstract sequence vectors and topological feature vectors as $128+64=192$ length vectors. Then, we feed them into the supervised learning model for downstream interaction identification and multi-family classification tasks. For the interaction identification task, the supervised learning model consists of one fully connected layer having 64 hidden units. The number of units in the output layer is decided by the number of classes we need to identify. For the protein interaction identification task, the class label is either 0 or 1 indicating interaction or non-interaction respectively. For the family classification task after the unsupervised learning phase, we build three-layer deep neural network(DNN) as shown in Fig. 3. We train the DNN for 200 epochs with a dropout rate at 0.5 and batch size at 64 . The number of units in the output layer is determined by the number of protein families we aim to categorize. 


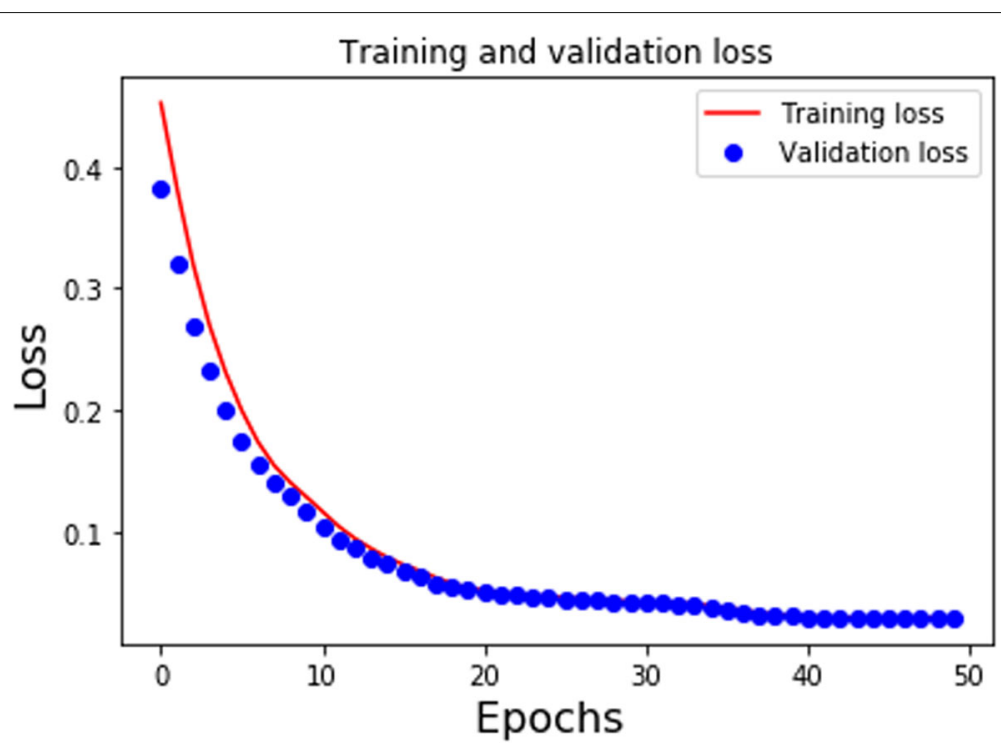

Fig. 4 SAE Loss for S.cerevisiae Species

\section{Evaluation Metrics}

During the experiments, we used Area Under the Receiver Operating Characteristic curve(AUC_ROC), Specificity(SPC), Accuracy(ACC), Precision, and Recall (or Sensitivity) to measure the prediction accuracy and data divergence using our method. The metric formulas are described as the following equations:

$$
\begin{aligned}
\text { Precision } & =\frac{T P}{T P+F P} \\
A C C & =\frac{T P+T N}{T P+T N+F P+F N} \\
S P C & =\frac{T N}{T N+F P} \\
\text { Recall } & =\frac{T P}{T P+F N}
\end{aligned}
$$

Here, TP, FP, TN, and FN denote True Positive, False Positive, True Negative and False Negative respectively.

\section{Comparison with traditional methods}

In our paper, we extensively compare our multi-modal deep representation learning framework with the traditional machine learning methods. We present the results of our method using all eight species in the DIP dataset and assess the receiver operating characteristics(ROC) scores using 5-Cross Validation methods in Fig. 5. Since the number of neurons in each hidden layer, the number of layers, and the vector size of the metapath representations of proteins are all critical parameters, we studied and tried various combinations to discover the model with the best performance. After that, the model with the best performance was selected to test the hold-out dataset as shown in Table 2. From the results in Fig. 5, we can see that most of the AUC scores achieved 0.99 using our model. To evaluate the performance of our method more thoroughly, we compared our model with traditional machine learning techniques[23, 24] including Nearest Neighbors $(k=2)$, Decision Tree, Random Forest and Naive Bayes in Figs. 6 and 7 respectively using ACC, Recall and AUC-ROC metrics.

\section{Comparison with state of the art methods}

We also compared our model over the DIP dataset across different species with the cutting-edge methods comprising of deep learning techniques using different evaluation metrics. Since previous researches use different species for evaluation, we compare them separately as shown

Table 1 Top-10 Similar Proteins to

DIP-41844N(5-hydroxytryptamine receptor 2A)

\begin{tabular}{lll}
\hline Protein ID & Protein Name & Cosine Similarity \\
\hline DIP-49960N & Nucleoside diphosphate kinase 3 & 0.90755 \\
DIP-31554N & Ribosomal protein S6 kinase alpha-3 & 0.89557 \\
DIP-38298N & $\begin{array}{l}\text { NADH dehydrogenase [ubiquinone] } \\
1 \text { beta subcomplex subunit 10 }\end{array}$ & 0.85293 \\
DIP-36377N & Microtubule-associated protein 1A & 0.82932 \\
DIP-61575N & Cannabinoid receptor 1 & 0.82696 \\
DIP-41406N & $\begin{array}{l}\text { Ankyrin repeat and sterile alpha motif } \\
\text { domain-containing protein 1B }\end{array}$ & 0.82623 \\
DIP-61135N & 39S ribosomal protein L28, & 0.82200 \\
& mitochondrial & \\
DIP-5723N & neurotrophin-3 receptor precursor & 0.81994 \\
DIP-61136N & Serum paraoxonase/arylesterase 2 & 0.81177 \\
DIP-59826N & Metabotropic glutamate receptor 2 & 0.79176 \\
\hline
\end{tabular}




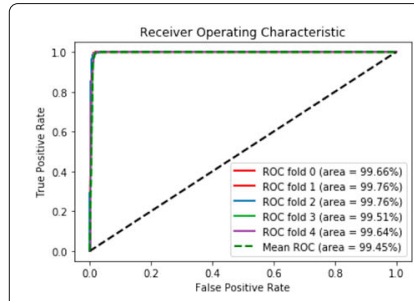

(a) C.elegans

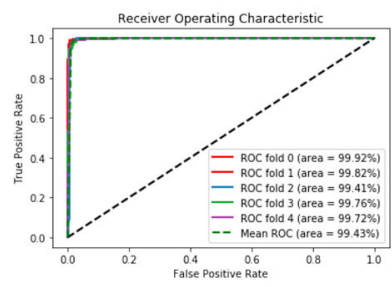

(e) S.cerevisiae

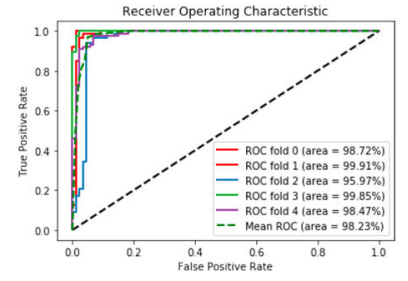

(b) C.elegans Multimodal

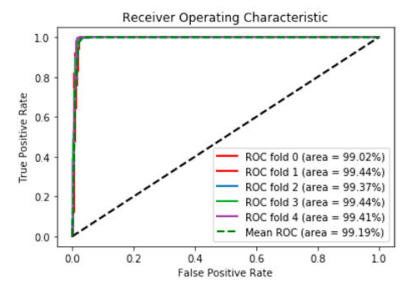

(f) S.cerevisiae Multimodal

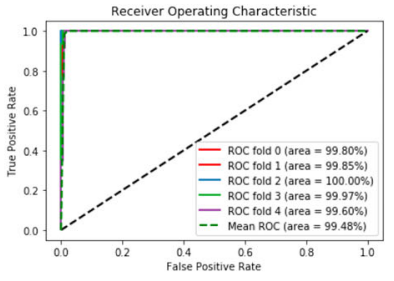

(c) H.sapiens

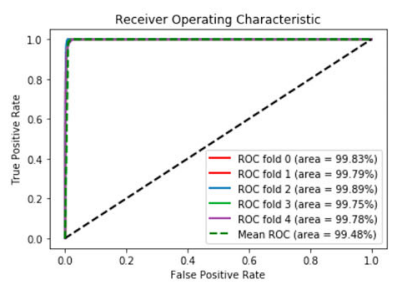

(g) H.pylori

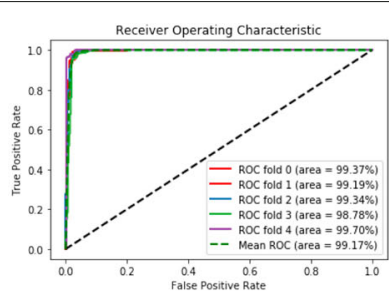

(d) H.sapiens Multimodal

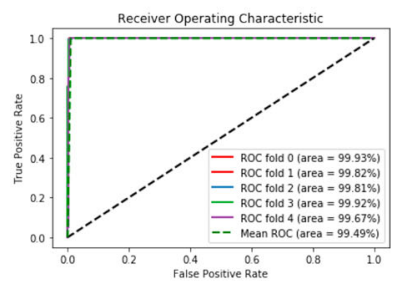

(h) H.pylori Multimodal

Fig. 5 5-CV AUC-ROC score for eight species

in Tables 3, 4, 5, 6, and 7. For instance, for S.cerevisiae species, compared to the other four most advanced methods, our multi-modal deep learning predictor still outperforms them. The ACC, Precision, Recall and AUC scores reach $2.76 \%, 4.27 \%, 5.73 \%$ and 0.0158 higher than Du's work, which proves the advantage of our model. And for E.coli, Drosophil and C.elegans datasets, we compare our model with two other most advanced methods [10] and [25] using the same metrics including Recall, ACC and SPC. For E.coli and C.elegans species, we outperform them using all three metrics. While, for Drosophil species we achieve higher performance using ACC and SPC metrics but slightly lower than other two methods using the Recall metric.

\section{Prediction Across Species}

We not only tested our model within the same species, but also used the S.cerevisiae training dataset as the overall training dataset and assess the prediction performance on the rest seven species using various metrics. In the experiment, the S.cerevisiae training dataset includes 36,006 negative and positive samples. The prediction performance of the rest seven species is presented in Table 8. We can see from the table that the accuracy for D. melanogaster, E. coli, C. elegans, $H$. sapiens, $H$. pylori, $M$. musculus, $R$. norvegicus are 96.76\%, 97.70\%, 98.44\%, 98.50\%, 98.84\%, 98.69\%, 99.77\%

respectively. Consequently, although only using single species training dataset, our multi-modal deep representation learning framework is still outperforms other methods using various evaluation metrics. Furthermore, we also examines D. melanogaster species and R. norvegicus species, which have not been explored by other methods yet and also achieves promising prediction accuracy.

\section{Prediction using HPRD dataset}

To compare other methods comprehensively and extending our previous work[29], we used HPRD as another benchmark dataset for testing. For the HPRD dataset, we only retrieved human proteins with family information from the Uni-Prot database while disgarded the human proteins without family annotations. After that, we have 16,915 human PPI interactions and 4185 human proteins. Then, we performed PPI prediction on the HPRD dataset. During this process, we generated the same amount of negative instances with positive samples using five subcellular positions consisting of Cyptoplasm, Endoplasimic reticulum, Golgi apparatus, Lysosome, Mitochondrion, Nucleus. The five cross validation ROC curve is plotted in

Table 2 Hyper-parameter settings

\begin{tabular}{ll}
\hline Parameter & Settings \\
\hline Batch Size & 64 \\
Learning Rate & 0.01 \\
Stacked Auto Encoder Architecture & $256-128-64-128-256$ \\
Optimization Method & SGD \\
Window size & 1 \\
CBOW Node Embedding Size & 128 \\
Neighboring Node & 4 \\
Metapath Length & 10 \\
\hline
\end{tabular}




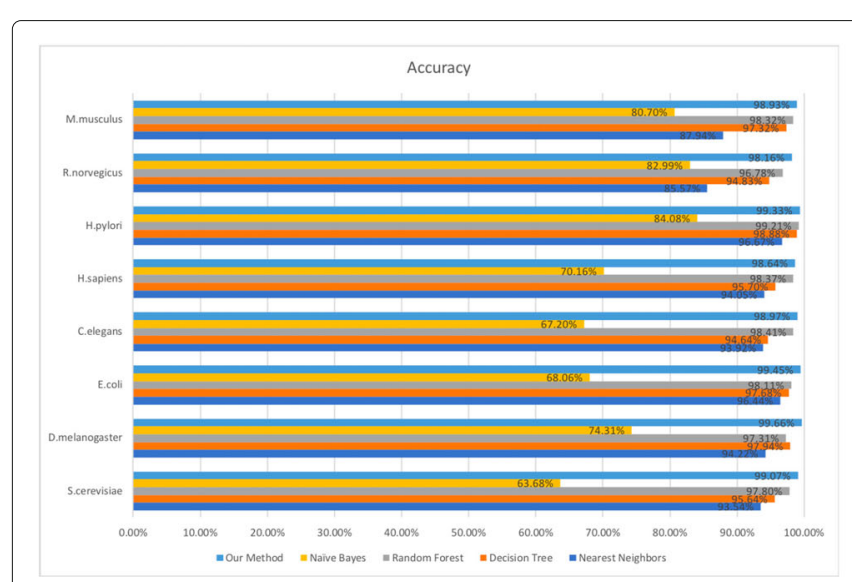

(a) Accuracy

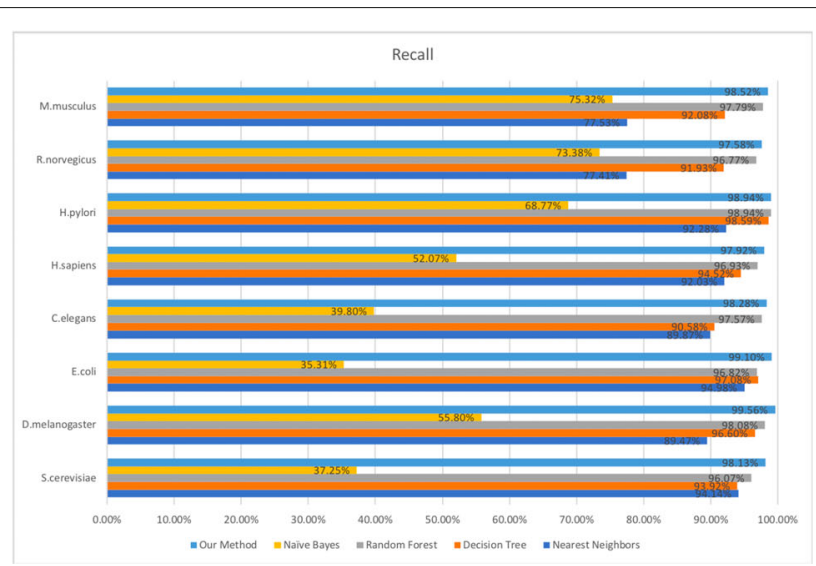

(b) Recall

Fig. $\mathbf{6}$ a Comparison of ACC score between our method and traditional machine learning techniques over eight species $\mathbf{b}$ Comparison of Recall score between our method and traditional machine learning techniques over eight species

Fig. 8. We compared our method with [10] and [30] using the same DIP H.sapiens dataset in Table 7 and the HPRD dataset and proved that our prediction accuracy is higher than those methods.

\section{Protein Family Classification}

In addition to interaction prediction, we performed downstream multi-family protein classification tasks as well using the same features from the unsupervised learning phase. In our experiments, family annotations are obtained from the UniProt database https://www.uniprot. $\mathrm{org} /$. We use all the proteins in the DIP dataset and acquire the families they belong to from the database. Amongst the protein families in the dataset, we only choose those families with more than 15 samples. This results in the top frequent 99 protein families to verify our results. We present the training accuracy and validation accuracy in Fig. 9 to show our model is not subjected to overfitting. Then, we evaluate using 5-CV and compare our prediction accuracy with traditional methods including Random Forest, SVC and GaussianNB classifiers. Since classifying proteins according to their family annotations is a multiclass classification task, we therefore use $\mathrm{F} 1$ score to assess the models' performance defined as the following Eq. 20:

$$
F 1=2 \times \frac{\text { precision } \times \text { recall }}{\text { precision }+ \text { recall }}
$$

As can be seen in Figs. 10 and 11, our multi-modal deep representation learning framework outperforms the

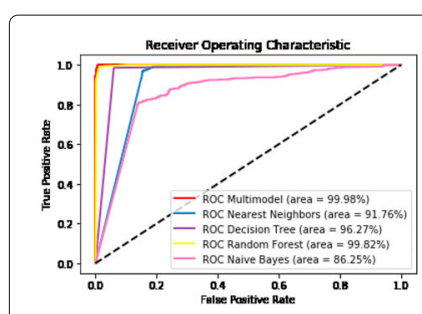

(a) C.elegans

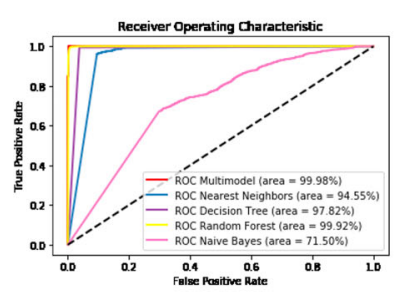

(e) D.melanogaster

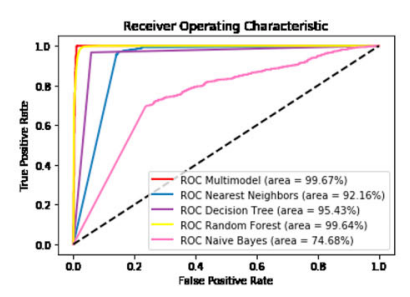

(b) H.sapiens

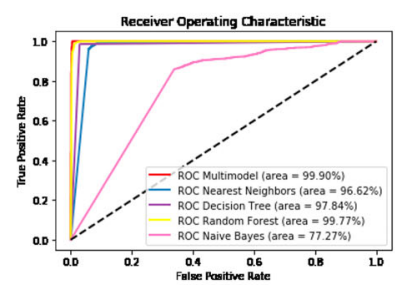

(f) E.coli

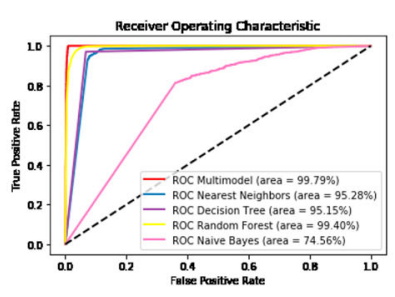

(c) S.cerevisiae

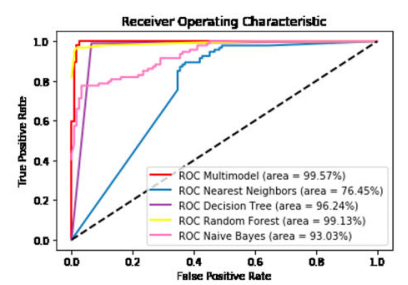

(g) R.norvegicus

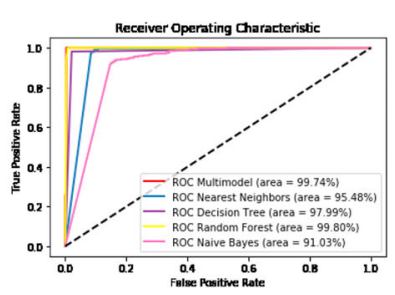

(d) H.pylori

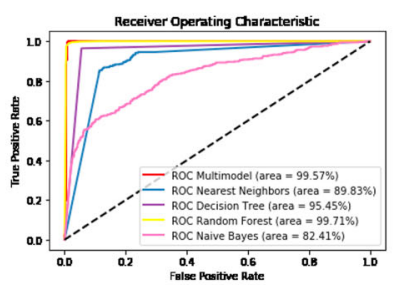

(h) M.musculus

Fig. 7 Comparison of the AUC-ROC score between our method and traditional machine learning methods over eight species 
Table 3 Comparison of 5-CV prediction performance between our method and state of the art methods using S.cerevisiae dataset(Note: N/A: Not Available)

\begin{tabular}{lllll}
\hline Method & Precision & ACC & Recall & AUC \\
\hline Our Method & $100.00 \% \pm 0.00 \%$ & $99.08 \% \pm 0.13 \%$ & $98.15 \% \pm 0.27 \%$ & $0.9908 \pm 0.13$ \\
Du's work[9] & $96.65 \% \pm 0.59 \%$ & $94.43 \% \pm 0.30 \%$ & $92.06 \% \pm 0.36 \%$ & 0.9754 \\
Wong's work[26] & $96.45 \% \pm 0.45 \%$ & $91.10 \% \pm 0.31 \%$ & $93.92 \% \pm 0.36 \%$ & $0.94 \pm 0.002$ \\
You's work[27] & $91.94 \% \pm 0.62 \%$ & $91.36 \% \pm 0.36 \%$ & $90.76 \% \pm 0.69 \%$ & $0.9707 \pm 0.12$ \\
Guo's work[3] & $88.87 \% \pm 6.61 \%$ & $89.33 \% \pm 2.67 \%$ & $87.37 \% \pm 0.22 \%$ & N/A \\
\hline
\end{tabular}

Table 4 Training performance between our method and other methods over the E.coli species(5-CV)

\begin{tabular}{llll}
\hline Method & Recall & ACC & SPC \\
\hline Our Method & $97.20 \%$ & $97.85 \%$ & $99.08 \%$ \\
Sun's work[10] & $96.89 \%$ & $96.05 \%$ & $95.28 \%$ \\
Guo's work[25] & $95.11 \%$ & $92.73 \%$ & $90.35 \%$ \\
\hline
\end{tabular}

Table 5 Training performance between our method and other methods over the Drosophil species(5-CV)

\begin{tabular}{llll}
\hline Method & Recall & ACC & SPC \\
\hline Our Method & $99.06 \%$ & $99.20 \%$ & $99.78 \%$ \\
Sun's work[10] & $99.51 \%$ & $97.84 \%$ & $96.28 \%$ \\
Guo's work[25] & $99.53 \%$ & $90.09 \%$ & $80.65 \%$ \\
\hline
\end{tabular}

Table 6 Training performance between our method and other methods over the C.elegans species(5-CV)

\begin{tabular}{llll}
\hline Method & Recall & ACC & SPC \\
\hline Our Method & $98.28 \%$ & $98.81 \%$ & $100.00 \%$ \\
Sun's work[10] & $99.35 \%$ & $97.23 \%$ & $95.28 \%$ \\
Guo's work[25] & $96.46 \%$ & $97.51 \%$ & $98.55 \%$ \\
\hline
\end{tabular}

Table 7 Prediction accuracy comparison between our method and state of the art methods over the H.sapiens and HPRD dataset

\begin{tabular}{lll}
\hline Method & HPRD & DIP \\
\hline Our Method & $97.61 \%$ & $95.94 \%$ \\
Sun's work[10] & $97.14 \%$ & $93.77 \%$ \\
Pan's work[30] & $86.70 \%$ & $90.04 \%$ \\
\hline
\end{tabular}

Table 8 Prediction Results on Seven Species using Our Proposed Framework, Based on S.cerevisiae Training Dataset as the Overall Training Dataset (Note: N/A: Not Available)

\begin{tabular}{|c|c|c|c|c|}
\hline Species & Our Method & Du's work[9] & Huang's work[28] & Zhou's work[2] \\
\hline C.elegans & $98.44 \%$ & $94.84 \%$ & $81.19 \%$ & $75.73 \%$ \\
\hline H.sapiens & $98.50 \%$ & $93.77 \%$ & $82.22 \%$ & $76.27 \%$ \\
\hline M.musculus & $98.69 \%$ & $91.37 \%$ & $79.87 \%$ & $76.88 \%$ \\
\hline H.pylori & $98.84 \%$ & $93.66 \%$ & $82.18 \%$ & N/A \\
\hline D.melanogaster & $96.76 \%$ & N/A & N/A & N/A \\
\hline E.coli & $97.70 \%$ & $92.19 \%$ & $66.08 \%$ & $71.24 \%$ \\
\hline R.norvegicus & $99.77 \%$ & N/A & N/A & N/A \\
\hline
\end{tabular}




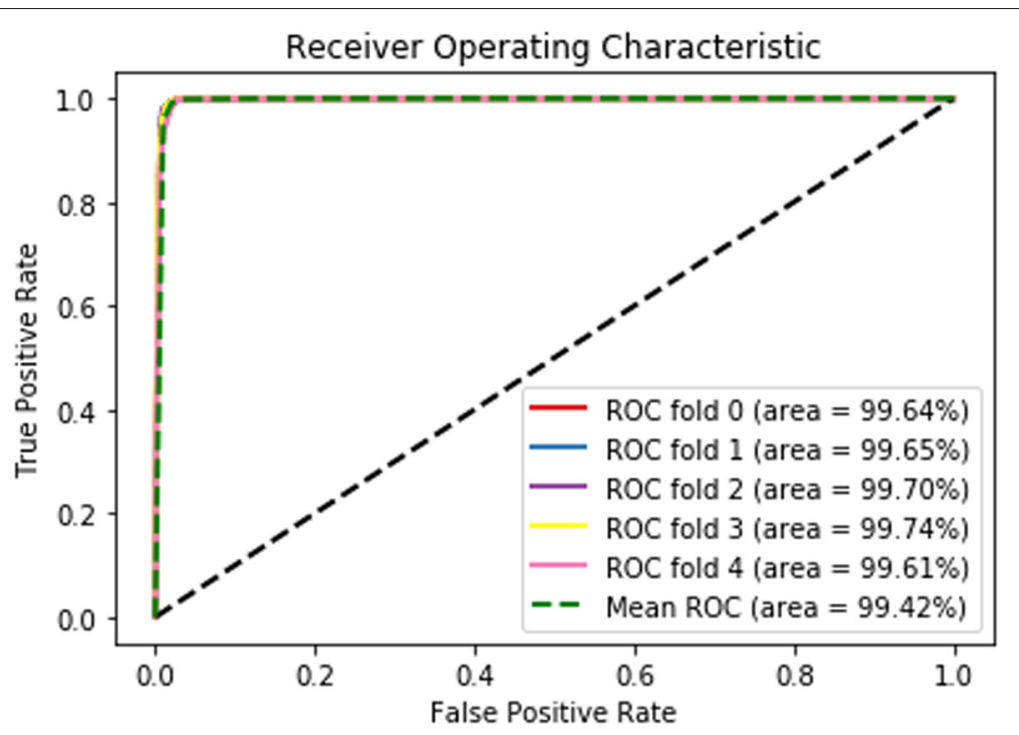

Fig. 8 5-CV AUC-ROC score for HPRD dataset

traditional methods using both Micro-F1 and Macro-F1 scores.

\section{Discussion}

In this paper, we compare our multi-modal deep learning framework with representative traditional machine learning methods and state of the art methods. These state of the art methods include deep learning methods. Then, we verify our method across all eight species provided by DIP_FULL and HPRD datasets. For instance, for the S.cerevisiae dataset in DIP, our accuracy achieved $99.79 \%$ while the performance of the other four methods was
95.28\%, 95.15\%, 99.40\% and $74.56 \%$ respectively. As for the Recall score for S.cerevisiae species, our recall scores achieved $98.13 \%$ while the values of the other four methods are $97.07 \%, 93.92 \%, 94.14 \%$ and $37.25 \%$ respectively. For the HPRD dataset, we can see from the results that the mean ROC score achieves 0.9942 , which is consistent with other species in the DIP dataset.

Additionally, we predict protein families based on the deep protein representation features with our models on the DIP dataset. Our method achieves up to $33.9 \%$ improvements in terms of Micro-F1 score and achieves up to $74.4 \%$ improvements in terms of Macro-F1 score over

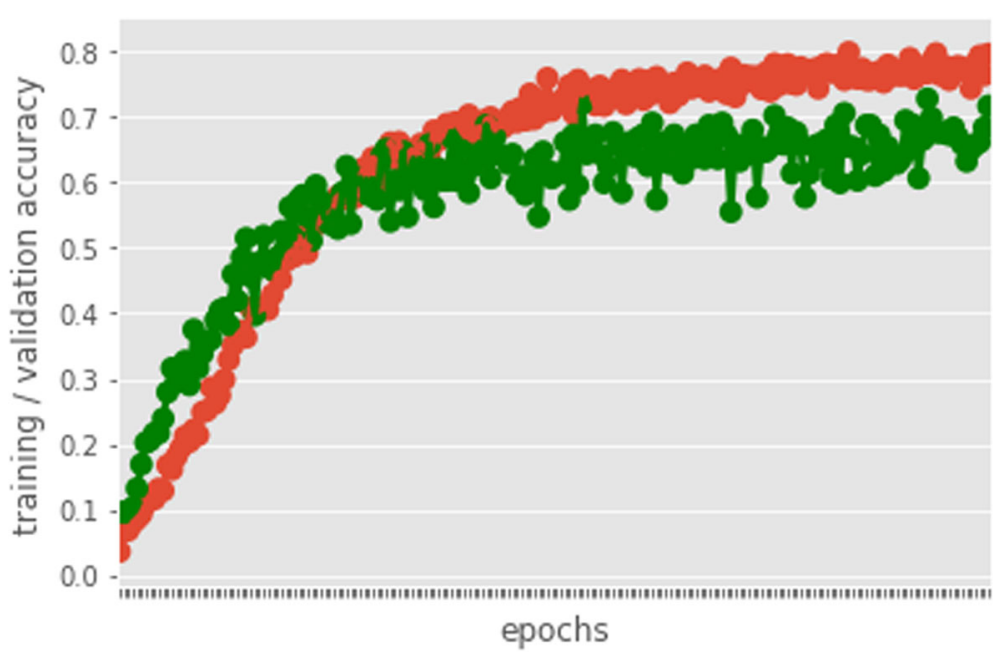

Fig. 9 Training and Validation Accuracy 


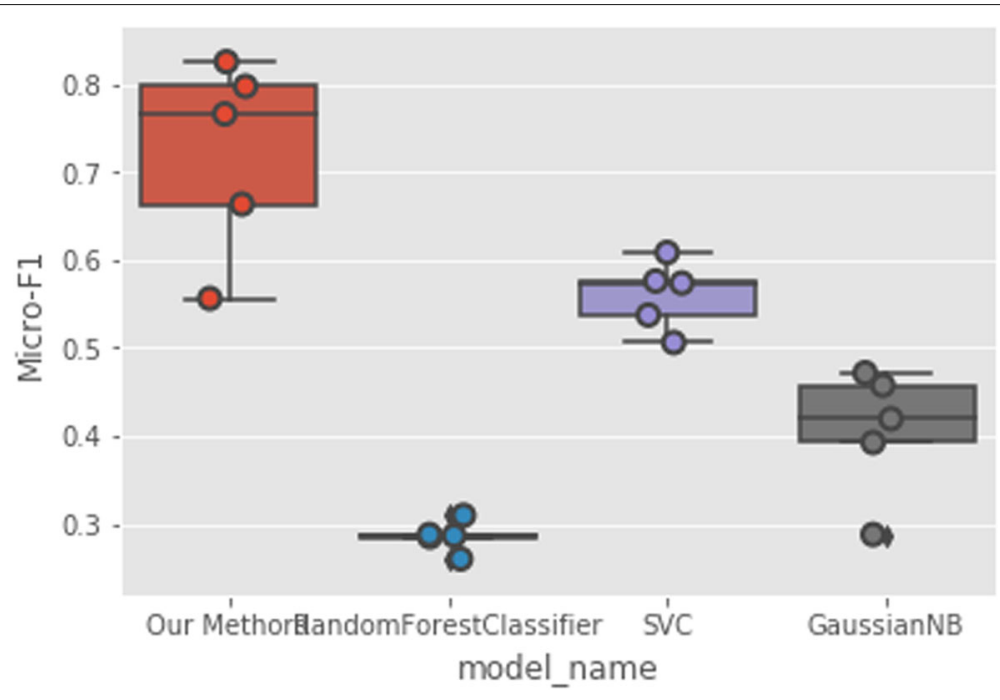

Fig. 10 Protein Multi-Family Classification Micro-F1 Score

the best performance among Random Forest, SVC and GaussianNB classifiers. Different from other protein family classification methods[31-33] which require at least 200 instances for each family, our method does not heavily rely on large dataset.

\section{Conclusions}

In summary, our multi-modal deep representation learning framework harvest features that are highly predictive of protein function. It captures both sequential protein raw information with the topological structure to improve the PPI prediction accuracy and multi-class classification accuracy given the complex, non-linear interaction networks PPI network. We apply our methods on both DIP and HPRD datasets. After applying the CBOW model based on generated metapaths, our model is able to take into account the graph topological information into account. We use various mainstream metrics to assess the performance over the new released DIP_20170205 FULL dataset including eight species and HPRD datasets. Through extensive comparisons with both traditional machine learning methods and state of the art deep learning methods, we prove that our method outperforms most of them over the same datasets.

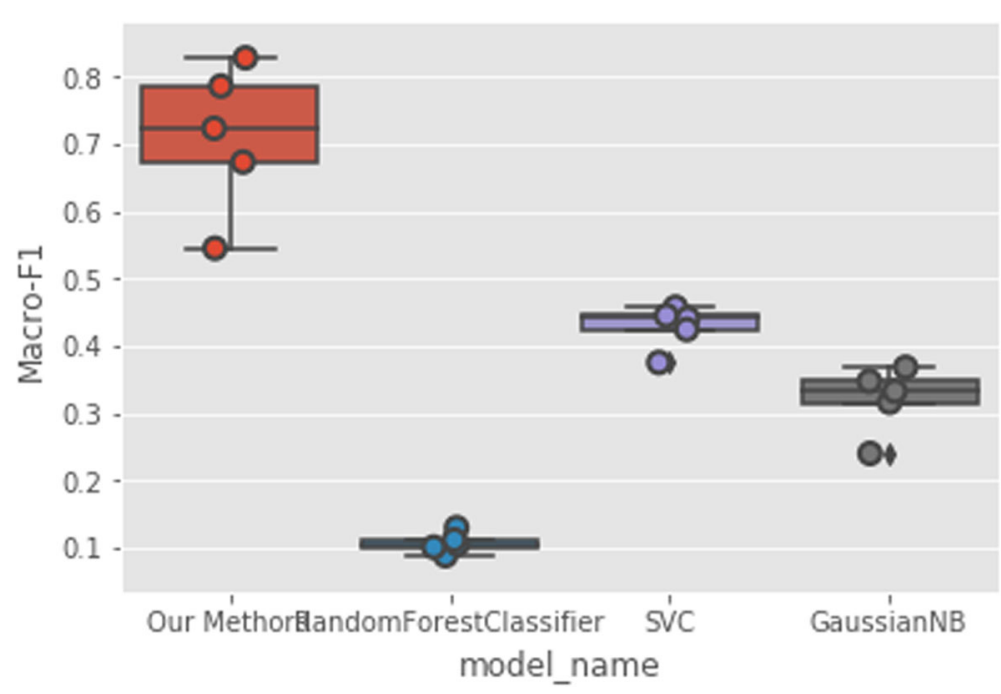

Fig. 11 Protein Multi-Family Classification Macro-F1 Score 


\section{Abbreviations}

AUC: Area under curve CBOW: Continuous bag-of-words; DNN: Deep neural networks; DT: Decision trees; FN: False negative; FP: False positive; NB: Naive bayes; NN:Nearest neighbor; ROC: Receiver operating characteristics; SAE: Stacked Auto Encoder; SGD: Stochastic gradient descent; TN: True negative; TP: True positive

\section{Acknowledgements}

Not applicable.

\section{About this supplement}

This article has been published as part of BMC Bioinformatics Volume 20 Supplement 16, 2019: Selected articles from the IEEE BIBM International Conference on Bioinformatics \& Biomedicine (BIBM) 2018: bioinformatics and systems biology. The full contents of the supplement are available online at https:// bmcbioinformatics.biomedcentral.com/articles/supplements/volume-20supplement- 16 .

\section{Authors' contributions}

DZ implemented the algorithms and analyzed the results. MK advised the research. DZ and MK wrote the manuscripts. Both authors read and approved of the final manuscript.

\section{Funding}

Publication costs were funded by grant R44CA206782 from the National Cancer Institute, part of the U.S. National Institutes of Health $(\mathrm{NIH})$.

\section{Availability of data and materials}

The DIP dataset is publicly available at http://dip.mbi.ucla.edu/dip/. The HPRD dataset is publicly available at http://www.hprd.org/. The UniProt dataset is publicly available at https://www.uniprot.org/locations/. The source code is available upon request.

\section{Ethics approval and consent to participate}

Not applicable.

\section{Consent for publication}

Not applicable.

\section{Competing interests}

The authors declare that they have no competing interests.

\section{Published: 2 December 2019}

\section{References}

1. Yang $L$, Xia J-F, Gui J. Prediction of protein-protein interactions from protein sequence using local descriptors. Protein Pept Lett. 2010;17(9): 1085-90.

2. Zhou YZ, Gao Y, Zheng YY. Prediction of protein-protein interactions using local description of amino acid sequence. Advanc Comput Sci Educ Appl. 2011254-62. https://doi.org/10.1007/978-3-642-22456-0_37.

3. Guo Y, Yu L, Wen Z, Li M. Using support vector machine combined with auto covariance to predict protein-protein interactions from protein sequences. Nucleic Acids Res. 2008;36(9):3025-30.

4. Creasey EA, Delahay R, Daniell SJ, Frankel G. Yeast two-hybrid system survey of interactions between lee-encoded proteins of enteropathogenic escherichia coli. Microbiology. 2003;149(8):2093-106. https://doi.org/10.1099/mic.0.26355-0.

5. Ho Y, Gruhler A, Heilbut A, Bader GD, Moore L, Adams S-L, Millar A, et al. Systematic identification of protein complexes in saccharomyces cerevisiae by mass spectrometry. Nature. 2002;6868:180.

6. Bhasin M, Raghava GP. Classification of nuclear receptors based on amino acid composition and dipeptide composition. J Biol Chem. 2004;279:23262-6.

7. Saidi R, Maddouri M, Nguifo EM. Protein sequences classification by means of feature extraction with substitution matrices. BMC bioinformatics. 2010;11(1):175.

8. Yann L, Bengio Y, Hinton G. Deep learning. nature. 2015;7553:436.

9. DuX, Sun S, Hu C, Yao Y, Yan Y, Zhang Y. Deepppi: boosting prediction of protein-protein interactions with deep neural networks. J Chem Inf Model. 2017;57(6):1499-510.
10. Sun $T$, Zhou B, Lai L, Pei J. Sequence-based prediction of protein protein interaction using a deep-learning algorithm. BMC bioinformatics. 2017;18(1):277.

11. Lee TK, Nguyen T. Protein family classification with neural networks. 2016. https://cs224d.stanford.edu/reports/LeeNguyen.pdf.

12. Peng $W$, Li $M$, Chen $L$, Wang L. Predicting protein functions by using unbalanced random walk algorithm on three biological networks. IEEE/ACM Trans Comput Biol Bioinforma. 2017;2:360-9.

13. Fa R, Cozzetto D, Wan C, Jones DT. Predicting human protein function with multi-task deep neural networks. PloS one. 2018;13(6):0198216.

14. Chen Z, Zhao P, Li F, Leier A, Marquez-Lago TT, Wang Y, Webb Gl, et al. ifeature: a python package and web server for features extraction and selection from protein and peptide sequences. Bioinformatics. 2018;1:4.

15. Lee TY, Lin ZQ, Hsieh S-J, Bretaña NA, Lu C-T. Exploiting maximal dependence decomposition to identify conserved motifs from a group of aligned signal sequences. Bioinformatics. 2011;27(13):1780-7.

16. Shen J, Zhang J, Luo X, Zhu W, Yu K, Chen K, Li Y, Jiang H. Predicting protein-protein interactions based only on sequences information. Proc Natl Acad Sci. 2007;104(11):4337-41.

17. Perozzi B, Al-Rfou R, Skiena S. Deepwalk: Online learning of social representations. Proc 20th ACM SIGKDD Int Conf Knowl Discov Data Min. 2014701-10. https://doi.org/10.1145/2623330.2623732.

18. Dong Y, Chawla NV, Swami A. metapath2vec: Scalable representation learning for heterogeneous networks. In: Proceedings of the 23rd ACM SIGKDD International Conference on Knowledge Discovery and Data Mining. ACM; 2017. p. 135-44.

19. Sun Y, Han J. Mining heterogeneous information networks: principles and methodologies. Synth Lect Data Min Knowl Discov. 2012;3(2):1-159. https://doi.org/10.2200/s00433ed1v01y201207dmk005.

20. Goyal P., Ferrara E. Graph embedding techniques, applications, and performance: A survey. arXiv. 2017;1705.02801:

21. Cao $S, L u W, X u Q$ Q. Deep neural networks for learning graph representations. In: Thirtieth AAAI Conference on Artificial Intelligence. 2016.

22. Hinton GE, Salakhutdinov RR. Reducing the dimensionality of data with neural networks. Science. 2006;313(5786):504-7.

23. Saha I, Zubek J, Klingström T, Forsberg S, Wikander J, Kierczak M, Maulik U, Plewczynski D. Ensemble learning prediction of protein protein interactions using proteins functional annotations. Mol BioSyst. 2014;10(4):820-30.

24. Martin S, Diana Roe D, Faulon J-L. Predicting protein-protein interactions using signature products. Bioinformatics. 2004;21 (2):218-26.

25. Guo Y, Li M, PuX, Li G, Guang X, Xiong W, Li J. Pred_ppi: a server for predicting protein-protein interactions based on sequence data with probability assignment. BMC research notes. 2010;3(1):145.

26. Wong L, You Z-H, Ming Z, Li J, Chen X, Huang Y-A. Detection of interactions between proteins through rotation forest and local phase quantization descriptors. Int J Mol Sci. 2015;17(1):21.

27. You H, Zhu L, Zheng C-H, Yu H-J, Deng S-P, Ji Z. Prediction of protein-protein interactions from amino acid sequences using a novel multi-scale continuous and discontinuous feature set. BMC Bioinformatics. 2014;15(15): 2014;15(15).

28. Huang Y-A, You Z-H, Gao X, Wong L, Wang L. Using weighted sparse representation model combined with discrete cosine transformation to predict protein-protein interactions from protein sequence. BioMed Res Int. 2015. https://doi.org/10.1155/2015/902198.

29. Zhang D, Kabuka MR. Multimodal deep representation learning for protein-protein interaction networks. IEEE Int Conf Bioinforma Biomed. 2018;Madrid Spain:. https://doi.org/10.1109/bibm.2018.8621366.

30. Pan $X Y$, Zhang $Y$, Shen HB. Large scale prediction of human protein protein interactions from amino acid sequence based on latent topic features. J Proteome Res. 2010;9(10):4992-5001.

31. Nguyen N-P, Nute M, Mirarab S, Warnow T, genomics BMC. Hippi: highly accurate protein family classification with ensembles of hmms. 2016;765. https://doi.org/10.1186/s12864-016-3097-0.

32. Szalkai B, Grolmusz V. Near perfect protein multi-label classification with deep neural networks. Methods. 2018;50-6. https://doi.org/10.1016/j. ymeth.2017.06.034

33. Naveenkumar KS, Mohammed BR, Vinayakumar HR, Soman KP. Protein family classification with neural networks. bioRxiv. 2018;414128.

\section{Publisher's Note}

Springer Nature remains neutral with regard to jurisdictional claims in published maps and institutional affiliations. 